\title{
Editorial
}

\section{The aims of middle ear surgery to improve hearing}

Times are changing in otology, not as they did in the sixties and seventies when microsurgical techniques developed, but rather with a growing realization that for a given surgical procedure the potential benefits are extremely varied. When the object of surgery is to improve the hearing, it is no longer sufficient to look at the patient's audiogram, identify an air-bone gap and suggest to the patient that by surgically closing the air bone gap their hearing will improve. In audiometric terms this will be so, but how much a patient will be satisfied with this improvement will vary and depends on many factors. It is relatively easy to suggest what these might be but how they interact is unknown. What requires to be developed is a means of assessing a patient's disability and desires before surgery. With the knowledge of the technical abilities of a given otologist doing a specific operation, the chances of the various outcomes following surgery can then be meaningfully discussed. Studies are currently underway to develop guidelines on how best to do this but in the meantime existing knowledge has to be used. The first thing every surgeon requires to know is how technically successful they are at performing middle ear surgery. This is learned by audit.

\section{Auditing technical success}

Provided the patient is capable of reliably responding, pure-tone audiometry carried out in a sound-proofed area with accurate masking, particularly for the bone-conduction thresholds, can hardly be bettered as a measure of the technical success of middle ear surgery to improve the hearing. Pathology in the middle ear attenuates the conduction of sound to the inner ear and any change in the air conduction thresholds is a measure of how much the conduction mechanism has been improved. The potential for improvement is assessed by the magnitude of the preoperative air-bone gap. Whether the full potential for improving the air conduction thresholds has been achieved can be assessed by looking at the magnitude of any residual air-bone gap.

Inevitably, as with all surgery, there are technical risks and inner ear damage with a resultant loss is the most likely in middle ear surgery. This most commonly occurs at the higher frequencies, $4 \mathrm{kHz}$ and above, but unfortunately can also affect the lower frequencies which are more important for speech. So monitoring of all thresholds, 0.25 to $8 \mathrm{kHz}$, should identify ears in which the inner ear has been damaged following surgery.

It is hoped that every otologist will periodically audit their surgery where they have hoped to improve the hearing so that they can state for each procedure (e.g. stapedotomy, malleus-incus reassembly), i) the percentage of patients in whom the air conduction thresholds would improve by certain magnitudes, ii) what the post-operative range in air-bone gaps would be for a given size of preoperative air-bone gap, and iii) in what percentage of patients a sensorineural loss would result.

This knowledge can then be used along with the other factors discussed below to advise future patients of the chances of achieving the various aims of surgery, it no longer being good enough to say, for example, to a patient that following stapedectomy there is a 90 per cent chance that your hearing will improve.

\section{The differing aims of surgery}

Most otologists will already use their previous experience to decide that it is worth operating on some patients whilst it is not on others. The criteria used to do so are informal and, of course, influenced by many factors. These include patient motivation, the surgeon's desire to operate or to train junior staff, and the availability of resources. However, even at the current state of the art there are patients in whom most would agree that a surgically-induced improvement in the hearing would be unlikely to bring benefit. If we take an adult patient with a modified radical mastoidectomy and a unilateral profound mixed impairment, the air conduction average being $90 \mathrm{~dB} \mathrm{HL}$, the bone conduction average $50 \mathrm{~dB} \mathrm{HL}$ and a normal contralateral ear. Few surgeons would suggest reconstructing the middle ear in such a patient with the primary aim of improving the hearing. This is because even if the air-bone gap were to be closed the patient would still have a moderate sensorineural impairment for which they would be unlikely to wear an aid because of the normal contralateral ear. From this example there is obviously a range of patients right through to those where surgery would be offered by most surgeons provided they had the technical ability. Such an example would be a patient with bilateral otosclerosis who declines a hearing aid and has air conduction thresholds of $50 \mathrm{~dB} \mathrm{HL}$ and bone conduction thresholds of $15 \mathrm{~dB}$ HL. In between these two examples there is a wide variety of combinations of pathology, levels of impairment, levels of bone conduction, degree of asymmetry and use of or acceptability of hearing aids that can be taken account. The last two factors are those least explored and tend to go unreported. Papers that state what the air and bone conduction thresholds are before and after surgery are common; papers that discuss how surgery has modified the use of hearing aids are rare. It is accepted that there are some difficulties in doing this but there is no reason why the degree of symmetry between ears should not be routinely reported and certainly be discussed with the patient before surgery. 


\section{(a) Degree of symmetry}

In a high proportion of patients with a conductive impairment, only one ear is affected and even if both ears are affected they are often unequally affected. In both circumstances the patient will have asymmetric hearing, there being a poorer and a better hearing ear. There are many aspects to binaural hearing including sound localization and separating out speech from a noisy background. However, we also know that it is possible to do each of these tasks with only one ear. The question then is how bad does the poorer hearing ear have to be for it not to take part in these listening processes. Surprisingly little is known about this because, as might be expected, the interaction between ears is complex. But let us make it simple because it is better to apply a simple rule of thumb rather than no rule at all.

Because the skull does not greatly block the transfer of sound around it, speech arising on the side of the poorer hearing ear will be heard primarily by the better hearing ear unless the hearing thresholds between ears are within $15 \mathrm{~dB}$. So for practical purposes, although the patient may have what they consider a better and poorer ear, they are only considered to have asymmetric hearing if the difference in average thresholds between ears is greater than $15 \mathrm{~dB}$. It is accepted practice that because of the albeit small risk of damage to hearing, surgery would initially be carried out on the poorer hearing ear. Thus before surgery, if the thresholds between ears are asymmetric, surgery will be carried out on the poorer ear. If after surgery they remain asymmetric and the thresholds are not brought to within $15 \mathrm{~dB}$ of each other, the patient is unlikely to notice a change in their hearing. This has been well established by many surgeons and particularly by the late Gordon Smyth who developed what he called the "Belfast rule of thumb" (Smyth and Paterson, 1985; Smyth, 1992). By asking patients to rate their perceived degree of success of the operation and comparing this with the degree of symmetry between the ears, he found that those least happy were those in whom the average air conduction difference between ears remained greater than $15 \mathrm{~dB}$.

The concept of comparing the degree of symmetry between ears before and after surgery has been developed in the Glasgow Benefit Plot (Browning, Gatehouse and Swan, 1991). Such a plot has considerable advantages in that as each patient in a series is illustrated it is possible to look at the results and apply varying definitions of asymmetry. Thus apart from being able to work out from the plot most of the conventional parameters of technical success, such as change in air conduction thresholds and closure of air-bone gaps, it is also possible to calculate in how many patients the ears are within $10,15,20$ etc $\mathrm{dB}$ of each other, before and after surgery.

However the results are reported, the greatest potential for lessening a patient's overall disability is in those in whom the hearing between ears pre-operatively is symmetric. Any improvement then in the air conduction thresholds will be noticed by the patient and reported as improved hearing. The magnitude of this improvement will primarily be related to the improvement in the air conduction thresholds in the better hearing ear which could be the non-operated ear before surgery and the operated ear after surgery. Again this is represented in the Glasgow Benefit Plot. What cannot be represented in such plots and is a major determinant of benefit is the change in use, if any, of hearing aids following surgery.

\section{(b) Hearing aid use}

The fact that a patient pre-operatively uses or could use a hearing aid is difficult to build into the current decisionmaking process. Hopefully, this will change as a result of future work, but at present there are some guidelines. In using these it is assumed that the hearing aid provision is optimum for a specific ear. If the air conduction thresholds improve following surgery there are three potential outcomes. The patient can i) dispense with aids altogether, ii) reduce the need to use them all the time or iii) gain greater benefit when wearing an aid in the operated ear.

It is usual to take $30 \mathrm{~dB} \mathrm{HL}$ as the upper limit of normal hearing and advise that if an individual's thresholds are better than that a hearing aid is unlikely to be of benefit. In considering surgery then, unless the bone conduction thresholds are better than $30 \mathrm{~dB} \mathrm{HL}$, the patient will still benefit from an aid even if the air-bone gap has been closed. It is also known that in general patients do not consult regarding amplification for their hearing until the thresholds in the better ear are $45 \mathrm{~dB}$ HL or worse (Lancet Editorial, 1987). So it could be argued that if surgery does not make the hearing thresholds in one ear better than $45 \mathrm{~dB} \mathrm{HL}$, the patient is likely to still wear a hearing aid the majority of the time. If the threshold in one ear lies between 30 and $45 \mathrm{~dB}$ HL then although a hearing aid would be of benefit in most situations, the disability when not wearing it is such that its use may become intermittent. Certainly in situations where use of an aid is not practical such as in bed or at a swimming pool, the patient will be materially better off.

In patients with a bilateral severe impairment (PTA > $70 \mathrm{~dB}$ HL) the type of impairment is almost certainly mixed (McClymont and Browning, 1991). Here the aim can only be to lessen the use of or to improve the benefit from an aid. Up until this point in the discussion, improvement in benefit from an aid has not been a major consideration, the difference in benefit from aiding an ear with a 45 as opposed to a $60 \mathrm{~dB}$ HL impairment being small. However, the difference in benefit from an aid in an ear with a $45 \mathrm{~dB}$ HL as opposed to one with an $80 \mathrm{~dB}$ HL impairment is considerable. This is primarily because the aid for the lesser impairment can be used at a lesser gain, hence causing less distortion and thus greater benefit. Thus surgery in individuals with bilateral mixed impairments will bring benefit if the air conduction thresholds in the aided ear can be made better than $70 \mathrm{~dB}$ HL.

\section{The way forward}

Today's patient with a potentially correctable conductive impairment should have a full discussion about the various possible outcomes with an otologist who knows his surgical potential from audit. The discussion will start with an assessment of the patient's current listening disability, how much it is a concern to them and their relative attitudes to surgery or a hearing aid. Fears of hospital and operations are often based on stories from the past but today's middle ear surgery can usually be done under local anaesthetic making a 24 hour hospital stay routine. Concepts of hearing aids are also often outdated and lack of benefit from these is often the view. This is not the case, as individuals with a conductive impairment almost invariably benefit from them though probably not as much as they would from technically successful surgery (Carlin et al. 1985). 
The patient's audiogram should then be considered in relation to the reported disability and in particular the degree of symmetry between ears. The potential aims of surgery can then be reasonably thought out and the chances of achieving them discussed with the patient. This will include a statement as to which is likely to be the better ear after surgery and how much residual disability they may expect in various circumstances. Whether a hearing aid will be of continued benefit will also be discussed.

It is accepted that some patients may find it too difficult to report their disability in detail and to make value judgements about the various options. There are others that will consider their surgeon to be marvellous no matter what happens. However, informed consent is necessary in everyone before surgery and this means that the objectives have been clearly stated and alternatives fully discussed at a level appropriate for each patient. This frequently requires to be in greater detail than might be initially imagined. Hopefully, in the near future, more objective measures will be developed to predict the comparative benefits of a hearing aid against surgery. When these become available, otologists will then be even more able to compliment the art of medicine, the skill of surgery with the veracity of science.

\section{References}

Browning, G. G., Gatehouse, S. , Swan, I. R. C. (1991) The Glasgow Benefit Plot: a new method for reporting benefits from middle ear surgery. Laryngoscope, 101:180-185.

Carlin, W. V., Lawson, E., Gatehouse, S., Browning, G. G. (1985) A comparison of hearing aid benefit in conductive and sensorineural hearing loss. Clinical Otolaryngology, 10: 114.

Editorial (1987) Hearing problems in elderly people: implications for services. Lancet, May 23, 1181-1182.

McClymont L. G., Browning, G. G. (1991) Characterization of severely and profoundly hearing impaired adults attending an audiology clinic. Journal of Laryngology and Otology, 105: 534-538.

Smyth, G. D. L., Patterson, C. C. (1985) Results of middle ear reconstruction: do patients and surgeons agree? American Journal of Otology, 6: 276-279.

Smyth, G. D. L. (1992) Toynbee Memorial Lecture 1992: Facts and fantasies in modern otology: the ear doctor's dilemma. Journal of Laryngology and Otology, 106: 591-596.

Professor G. G. Browning, M.D., F.R.C.S.,

Department of Otolaryngology,

Royal Infirmary,

Glasgow G4 0SF. 